\title{
Índice calcio-creatinina en una muestra matutina de orina para la estimación de hipercalciuria asociada con hematuria no glomerular observada en las edades infanto-juveniles
}

\author{
Susana Quiñones-Vázquez, María del Rosario Liriano-Ricabal, Sergio Santana-Porbén* \\ y José Reinaldo Salabarría-González \\ Departamento de Estudios de la Función Renal, Servicio de Laboratorio Clínico, Hospital Pediátrico Docente Juan Manuel Márquez, La Habana, Cuba
}

\begin{abstract}
Resumen
Introducción: La hipercalciuria suele revelarse durante el diagnóstico diferencial de la hematuria que acompaña a la litiasis renal. La exactitud diagnóstica de la excreción urinaria de calcio puede afectarse por las insuficiencias asociadas con la colección de orina de 24 horas. En este estudio se evaluó la utilidad diagnóstica del índice calcio/creatinina (ICaCre) en la estimación de la hipercalciuria asociada con hematuria y litiasis renal. Método: Se calculó el ICaCre de las concentraciones urinarias de calcio (mmol/l) y creatinina ( $\mu$ mol/l) en una alícuota de colección de 24 horas de orina en 169 niños y adolescentes atendidos por hematuria no glomerular (HNG) o litiasis renal (LR). La calciuria de 24 horas > $4.0 \mathrm{mg} / \mathrm{kg}$ en 24 horas se distribuyó según la presencia de HNG o LR. Resultados: El ICaCre promedio fue de $0.2 \pm 0.1 \mathrm{mg} / \mathrm{mg}$. La excreción urinaria de calcio estimada del ICaCre fue significativamente superior a la obtenida en colección de orina de 24 horas ( $p$ < 0.05). Los métodos de determinación de la calciuria concordaron en la frecuencia de hipercalciuria (ICaCre $39.5 \%$ vs. colección de 24 horas 32.1\%; $p>0.05$ ). Según la presencia de HNG o LR, la hipercalciuria se distribuyó de la siguiente manera: no HNG + no LR: 59\%; no HNG + LR: 60\% (diferencia: +1.0\%); HNG + no LR: 68.2\% (diferencia: $+9.2 \%) ; H N G+$ LR: $73.3 \%$ (diferencia: $+14.4 \%$ ). Conclusiones: El ICaCre para estimar la excreción urinaria de calcio puede ser efectivo en el estudio de la hipercalciuria asociada con HNG y LR.
\end{abstract}

Palabras clave: Hipercalciuria. Orina. Hematuria. Litiasis renal.

\section{Calcium-creatinine ratio in a morning urine sample for the estimation of hypercalciuria associated with non-glomerular hematuria observed in children and adolescents}

\section{Abstract}

Background: Hypercalciuria might be revealed during the differential diagnosis of hematuria accompanying renal lithiasis $(R L)$. In spite of this, diagnostic accuracy of calcium urinary excretion might be affected by incomplete 24-hour urine collections. In the present study, the diagnostic utility of calcium/creatinine (ICaCre) index for determining hypercalciuria associated with non-glomerular hematuria (NGH) and RL was assessed. Method: $/ \mathrm{CaCre}(\mathrm{mg} / \mathrm{mg})$ index was calculated from calcium DOI: 10.24875/BMHIM.M18000006
Disponible en internet: 02-03-2018 Bol Med Hosp Infant Mex. 2018;75:41-48 www.bmhim.com

ervados. 
(mmolll) and creatinine ( $\mu$ molll) concentrations in an aliquot from a 24-hour urine collection in 169 children and adolescents with NGH or RL. Calciuria values $>4.0 \mathrm{mg} / \mathrm{kg}$ in 24 hours were distributed according to the presence of NGH or RL. Results: Mean ICaCre index was $0.2 \pm 0.1 \mathrm{mg} / \mathrm{mg}$. Calciuria values estimated from ICaCre were statistically higher to those from 24-hour urine collection $(p<0.05)$. The frequency of hypercalciuria was independent from the measurement method (estimated from ICaCre $39.5 \%$ vs. $24 \mathrm{~h}$ collection $32.1 \%$; $p$ > 0.05). Hypercalciuria distribution was as follows: no NGH + no RL: $59.0 \%$; no $N G H+R L: 60.0 \%(\Delta=+1.0 \%) ; N G H+$ no $R L: 68.2 \%(\Delta=+9.2 \%) ; N G H+R L: 73.3 \%(\Delta=+14.4 \%)$. Conclusions: The use of ICaCre index for determining calcium urine excretion might be effective in the study of hypercalciuria associated with NGH and RL.

Key words: Hypercalciuria. Urine. Hematuria. Kidney lithiasis.

\section{Introducción}

La hipercalciuria es un hallazgo frecuente en los niños y adolescentes que acuden a un servicio de nefrología, debida a hematuria de causa no glomerular o a litiasis renal ${ }^{1,2}$. Sin embargo, podría tener otras causas, como la absorción intestinal aumentada del calcio dietético en respuesta a la actividad de la vitamina $D$ o la paratohormona, el hiperparatiroidismo primario y la falla tubular renal $^{3,4}$. La hipercalciuria idiopática ha sido identificada durante décadas como una causa importante de excreción urinaria de calcio aumentada en presencia de concentraciones séricas preservadas de este mineral ${ }^{4,5}$. Recientemente se ha descrito una forma de hipercalciuria dentro del contexto del «síndrome por exceso de prostaglandinas PGE1 y PGE2»6, por lo que las connotaciones diagnósticas y terapéuticas de la hipercalciuria obligan a discutir el estado actual de la exactitud de la determinación del calcio urinario.

El estudio de la excreción urinaria de una sustancia específica ha tenido siempre la dificultad inherente a la obtención de una colección de orina que sea «exacta», esto es, que sea representativa del periodo natural en que ocurre la excreción del soluto ${ }^{7}$. Tradicionalmente, este periodo natural se refiere a 24 horas en la vida del paciente; es por esta razón que la estimación de la excreción urinaria del soluto conlleva la recogida de la orina que se excreta a lo largo de 24 horas $^{8}$. Actualmente, cuando mucho, solo la tercera parte de las colecciones de 24 horas de orina reúnen las condiciones para denotarlas como «exactas»9. Se introducen, entonces, sesgos notables en el estimado de la excreción urinaria del analito de interés, y por extensión se afecta la calidad de la información que entrega el laboratorio 8,9 .

El índice de excreción de una sustancia (la cantidad de la sustancia en cuestión que se encuentra en una muestra de orina tomada en cualquier momento del día, una vez corregida para la cantidad coexcretada de creatinina) puede ser aceptado como un estimado confiable de la excreción urinaria de esa sustancia ${ }^{10}$. En virtud de ello, el índice calcio-creatinina (ICaCre) ha sido empleado para el diagnóstico de la hipercalciuria $^{11,12}$. Asimismo, se han descrito los valores esperados del ICaCre para diferentes poblaciones pediátricas $^{13}$; no obstante, este índice ha sido visto, cuando más, como una herramienta aplicable solo en el análisis de los casos de excreción aumentada de calcio ${ }^{14}$, por lo que las cantidades excretadas de este mineral deberían ajustarse a la duración de un día en la vida del sujeto mediante el ensayo de una colección de orina de 24 horas si se comprobara un ICaCre aumentado ${ }^{15}$.

El ICaCre obtenido con una muestra matutina de orina puede escalarse hasta un estimado confiable de la excreción urinaria de calcio (calciuria) de 24 horas, una vez corregido según la talla y la superficie corporal del sujeto mediante un modelo determinístico apropia$\mathrm{do}^{16}$. Un estimado de la calciuria de 24 horas a partir del ICaCre también permitiría explotar un único punto de corte para el diagnóstico de hipercalciuria, lo que aseguraría la comparabilidad de los resultados de estudios de esta condición completados en distintas locaciones geográficas.

En virtud de todo lo anterior, el objetivo de este trabajo fue determinar la exactitud de la calciuria de 24 horas cuando se estima a partir del ICaCre en un niño o adolescente que se presenta con hematuria no glomerular.

\section{Métodos}

Se realizó un estudio de tipo prospectivo, analítico. Fueron elegibles para participar los niños y adolescentes de cualquier sexo, con edades entre 1 y 18 años, que concurrieron en su momento a la institución para el 
diagnóstico de hematuria no glomerular, y que fueron admitidos en el Departamento de Estudios de la Función Renal (DEFR) para la determinación de la excreción urinaria de calcio en respuesta a pedidos de las consultas ambulatorias especializadas de nefrología, urología y nutrición, y las salas de hospitalización.

De cada uno de los participantes se registraron el sexo (masculino/femenino), la edad, los diagnósticos de hematuria no glomerular (presente/ausente) y litiasis renal (presente/ausente), junto con la talla $(\mathrm{cm})$ y el peso corporal $(\mathrm{kg})$. La hematuria no glomerular se estableció ante la presencia de hematíes no dismórficos en la muestra matutina de orina y la ausencia de sintomatología propia de las glomerulopatías (como edemas, proteinuria de rango nefrótico e hipertensión arterial).

Los datos demográficos, clínicos, antropométricos y bioquímicos de los niños y adolescentes incluidos en la serie de estudio se ingresaron en los registros digitales del DEFR mediante la aplicación NEFROCALC, construida sobre el programa Access para Office de Windows (Microsoft, Redmond, VA, EE.UU.) ${ }^{17}$.

\section{Obtención de las muestras de orina}

De cada participante se obtuvo una colección de orina de 24 horas. Se instruyó a los padres del paciente para que la emisión matutina de orina en el día de la entrega fuera colectada de forma separada para la construcción del ICaCre. Posteriormente, esta emisión se reintegró a la colección de 24 horas, una vez separada la alícuota para las determinaciones bioquímicas.

Las concentraciones urinarias de creatinina (OCre, $\mu \mathrm{mol} / \mathrm{l})$ y calcio $(\mathrm{OCa}, \mathrm{mmol} / \mathrm{l})$ se determinaron mediante un modo cinético de la reacción de Jaffé y después de la formación de un complejo coloreado con arsenazo III, respectivamente ${ }^{18,19}$, según los procedimientos analíticos locales vigentes. Los métodos analíticos empleados se encuentran implementados en un autoanalizador COBAS (Hoffman-La Roche, Francia).

\section{Determinación de la excreción observada de la sustancia}

La excreción urinaria de la sustancia en 24 horas se obtuvo de las cantidades presentes de la misma en la colección de orina:

Creatinina $(\mathrm{mg} / 24 \mathrm{~h})=$ OCre $(\mu \mathrm{mo} / /) \times 0.113 \times$ volumen $(/ / 24 \mathrm{~h}) \quad[1]$

Calcio $(\mathrm{mg} / 24 \mathrm{~h})=\mathrm{OCa}(\mathrm{mmol} / \mathrm{l}) \times 40 \times$ volumen $(\mathrm{l} / 24 \mathrm{~h}) \quad$ [2]

\section{Cálculo del índice calcio-creatinina}

La OCre y la OCa se emplearon en el cálculo del ICaCre con la siguiente ecuación:

$$
\text { ICaCre }(\mathrm{mg} / \mathrm{mg})=\frac{\mathrm{OCa}(\mathrm{mmol} / \mathrm{I}) \times 40}{\mathrm{OCre}(\mathrm{mmol} / \mathrm{I}) \times 0.113}
$$

\section{Estimación de la excreción urinaria de calcio}

La OCa se estimó mediante una ecuación propietaria desarrollada en el DEFR ${ }^{16}$ :

Calciuria $(\mathrm{mg} / 24 \mathrm{~h})=\mathrm{ICaCre} \times 0.5 \times$ talla $\times \mathrm{SC} \times 8.333$ [4]

donde SC es la superficie corporal $\left(\mathrm{m}^{2}\right)$ calculada mediante la ecuación propuesta por DuBois y DuBois ${ }^{20}$. Por su parte, el número 0.5 corresponde a una constante de proporcionalidad que describe la generación endógena de creatinina del niño/adolescente. Asimismo, el número 8.333 representa una constante de escalamiento métrico empleada para convertir las unidades del ICaCre en $\mathrm{mg} / 24 \mathrm{~h}$.

La excreción urinaria de calcio se calificó como patológicamente elevada si la calciuria medida/estimada fue $>4.0 \mathrm{mg} / \mathrm{kg}$ en 24 horas. Los valores estimados del $\mathrm{ICaCre}$ de calciuria de 24 horas fueron desagregados según la presencia de hematuria no glomerular o de litiasis renal.

\section{Aseguramiento de la calidad de la colección de orina de $\mathbf{2 4}$ horas}

El completamiento de las colecciones de orina de 24 horas se calificó como adecuado mediante la ecuación desarrollada por Ghazali, et al. ${ }^{21,22}$.

\section{Procesamiento de los datos y análisis estadístico}

Los datos recuperados de los pacientes incluidos en la serie de estudio fueron reducidos hasta estadígrafos de locación (media), dispersión (desviación estándar) y agregación (frecuencias absolutas/relativas, porcentajes), según el tipo de la variable. Se empleó para ello la hoja de cálculo electrónica Excel para Office de Windows (Microsoft, Redmond, VA, EE.UU.).

Los valores de calciuria de 24 horas se filtraron según el completamiento de la colección de orina de 24 horas (adecuada o inadecuada) y la naturaleza de la calciuria de 24 horas (no patológica o patológica).

La existencia de diferencias entre los valores observados y predichos de la calciuria de 24 horas se 
examinó mediante una prueba de comparación de medias apareadas basado en la distribución $t$ de Student $^{23}$. La comparabilidad entre los valores observados y predichos de la calciuria de 24 horas se estimó mediante técnicas de regresión lineal basadas en los mínimos cuadrados ${ }^{23}$. El coeficiente $r^{2}$ de determinación se obtuvo como un indicador de la exactitud analítica de la ecuación predictiva de la calciuria de 24 horas. La pendiente $b$ de la recta de comparación de métodos sirvió para estimar la correspondencia entre ambos métodos $^{23}$

Los estudios de comparación de métodos se complementaron con la construcción del gráfico Bland-Altman para las diferencias entre la calciuria de 24 horas medida en la colección de orina y estimada del ICaCre. Se construyeron límites de control al 95\% para la diferencia entre los valores anticipando una colección adecuada de orina de 24 horas. Se contabilizaron los valores estimados de la calciuria de 24 horas incluidos dentro de los límites de control.

Adicionalmente, la concordancia diagnóstica entre los valores observados y predichos de la calciuria de 24 horas se examinó mediante la prueba de McNemar para observaciones apareadas basada en la distribución $\chi^{2}$ y la prueba de Kappa. En todo momento se utilizó un nivel de significación del $5 \%{ }^{23}$.

\section{Resultados}

La serie de estudio quedó constituida por 169 niños y adolescentes que cumplieron los criterios de inclusión de la investigación. La tabla 1 muestra las características demográficas, clínicas y antropométricas de los participantes. Como puede observarse, la serie de estudio se repartió equitativamente entre varones y hembras. La edad promedio fue de $10.4 \pm 3.7$ años, con predominio del grupo de edad de entre 5 y 12 años. La hematuria se presentó en el $21.9 \%$ de la serie de estudio. Por otro lado, la litiasis renal prevaleció en el $11.1 \%$ de los niños y adolescentes estudiados. El índice de masa corporal promedio fue de $18.1 \pm 3.3 \mathrm{~kg} /$ $\mathrm{m}^{2}$. Por su parte, la superficie corporal promedio fue de $1.2 \pm 0.3 \mathrm{~m}^{2}$.

La tabla 2 muestra las características de las determinaciones hechas en las muestras de orina. El volumen promedio de la colección de orina de 24 horas fue de $923.3 \pm 500.9 \mathrm{ml}$. La creatininuria de 24 horas fue de $16.8 \pm 8.0 \mathrm{mg} / \mathrm{kg}$. El $47.9 \%$ de las colecciones de orina de 24 horas fueron adecuadas.

La calciuria de 24 horas, estimada con las ecuaciones 3 y 4 , fue significativamente mayor que la medida
Tabla 1. Características demográficas, clínicas y antropométricas de los niños y adolescentes incluidos en la serie de estudio

\begin{tabular}{|l|c|}
\hline Característica & n (\%) \\
\hline $\begin{array}{l}\text { Sexo } \\
\text { Masculino } \\
\text { Femenino }\end{array}$ & $86(50.9)$ \\
\hline Edad (años) & $83(49.1)$ \\
\hline Grupo de edad & $10.4 \pm 3.7$ \\
\hline $\begin{array}{l}\text { Preescolares (1-4 años) } \\
\text { Adolescentes }(\geq 13 \text { años) }\end{array}$ & $7(4.1)$ \\
\hline Hematuria & $99(58.6)$ \\
\hline Litiasis renal & $63(37.3)$ \\
\hline Talla (cm) & $37(21.9)$ \\
\hline Peso $(\mathrm{kg})^{*}$ & $20(11.1)$ \\
\hline Índice de masa corporal $\left(\mathrm{kg} / \mathrm{m}^{2}\right)^{*}$ & $142.4 \pm 19.1$ \\
\hline Superficie corporal $\left(\mathrm{m}^{2}\right)^{*}$ & $38.2 \pm 14.5$ \\
\hline
\end{tabular}

*Media \pm desviación estándar.

Fuente: registros del estudio $(\mathrm{N}=169)$.

Tabla 2. Características bioquímicas de las determinaciones hechas en las colecciones de orina

\begin{tabular}{|c|c|}
\hline Característica & n (\%) \\
\hline Volumen $(\mathrm{ml})^{*}$ & $923.3 \pm 500.9$ \\
\hline Creatinina $\left(\mu \mathrm{mol} / \mathrm{I}^{*}\right.$ & $6740.2 \pm 3488.2$ \\
\hline Creatininuria $(\mathrm{mg} / 24 \mathrm{~h})^{*}$ & $638.6 \pm 405.1$ \\
\hline Creatininuria $(\mathrm{mg} / \mathrm{kg} \text { en } 24 \mathrm{~h})^{*}$ & $16.8 \pm 8.0$ \\
\hline Colecciones adecuadas & $81(47.9)$ \\
\hline Calcio $(\mathrm{mmol} / \mathrm{l})^{*}$ & $3.3 \pm 2.4$ \\
\hline $\begin{array}{l}\text { Calciuria (mg) medida en una colección de } \\
24 \mathrm{~h}^{*}\end{array}$ & $108.1 \pm 84.8$ \\
\hline $\begin{array}{l}\text { Calciuria }(\mathrm{mg} / \mathrm{kg}) \text { medida en una colección } \\
\text { de } 24 \mathrm{~h}^{*}\end{array}$ & $3.0 \pm 2.3$ \\
\hline $\begin{array}{l}\text { Calciuria (> } 4.0 \mathrm{mg} / \mathrm{kg} \text { ) medida en una } \\
\text { colección de } 24 \mathrm{~h}\end{array}$ & $129(76.3)$ \\
\hline $\begin{array}{l}\text { Índice } \mathrm{Ca} / \mathrm{Cre} \text { medido en una muestra } \\
\text { matutina de orina* }\end{array}$ & $0.19 \pm 0.13$ \\
\hline $\begin{array}{l}\text { Calciuria }(\mathrm{mg} / 24 \mathrm{~h}) \text { estimada mediante la } \\
\text { ecuación propietaria* }\end{array}$ & $135.9 \pm 98.7$ \\
\hline $\begin{array}{l}\text { Calciuria (mg/kg en } 24 \text { h) estimada } \\
\text { mediante la ecuación propietaria* }\end{array}$ & $3.8 \pm 2.6$ \\
\hline $\begin{array}{l}\text { Calciuria (> } 4.0 \mathrm{mg} / \mathrm{kg} \text { en } 24 \mathrm{~h} \text { ) estimada } \\
\text { mediante la ecuación propietaria }\end{array}$ & $104(61.5)$ \\
\hline
\end{tabular}




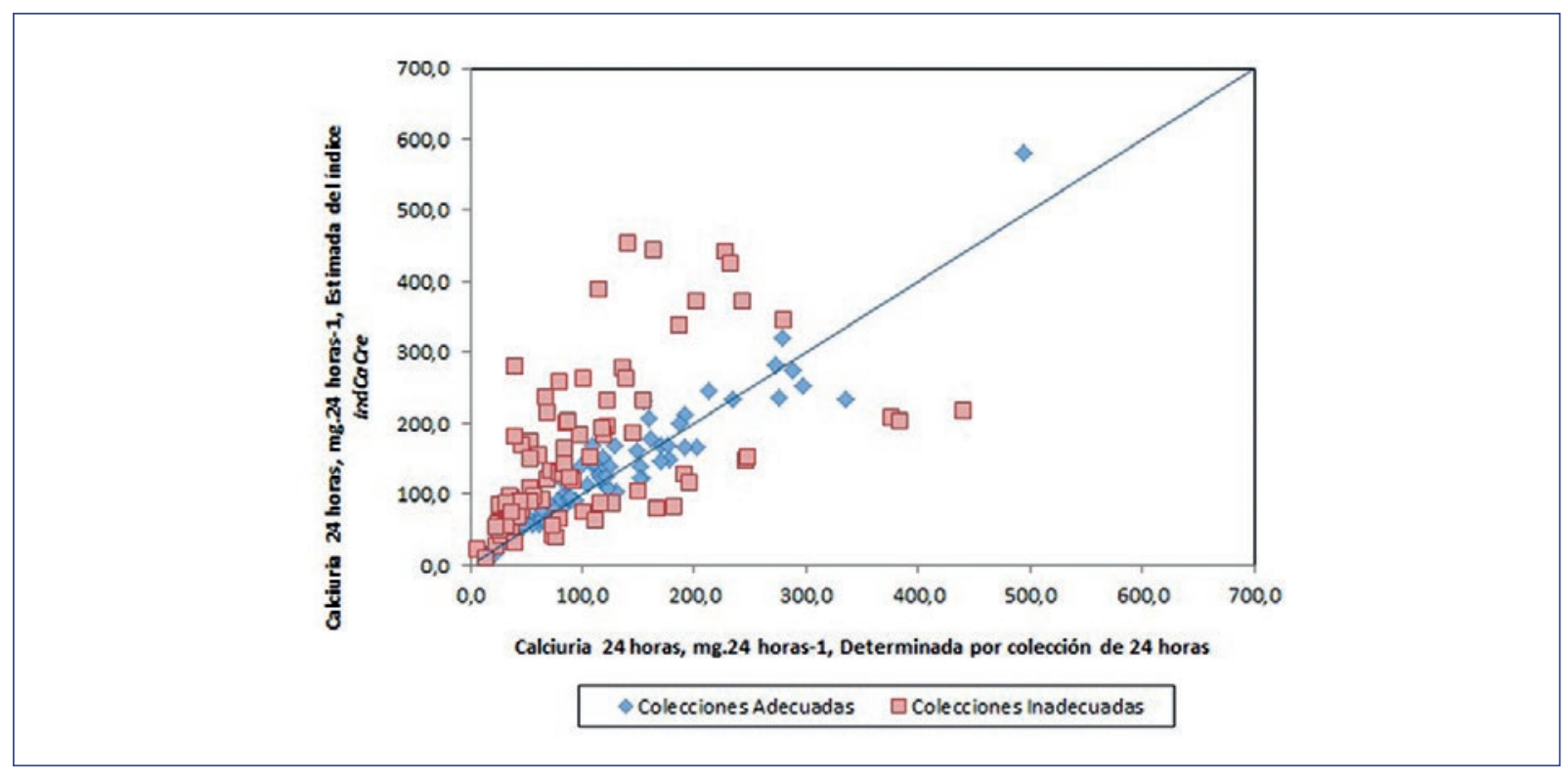

Figura 1. Relación entre la calciuria de 24 horas estimada mediante las fórmulas propietarias desarrolladas en este trabajo y la medida tras la recolección de orina de 24 horas. Recta de comparación de métodos entre los valores observados y estimados de la calciuria de 24 horas. Colecciones adecuadas de orina: calciuria estimada 24 horas = $9.3+0.97^{*}$ (calciuria observada en 24 horas: coeficiente $r^{2}=0.91$; error típico de la regresión $=26.0$ ) vs. colecciones inadecuadas, calciuria estimada 24 horas $=79.8+0.69^{*}$ (calciuria observada 24 horas: coeficiente $r^{2}=0.29$; error típico de la regresión $=90.3$ ). En ambas instancias, la probabilidad de ocurrencia de la pendiente $p<0.05$.

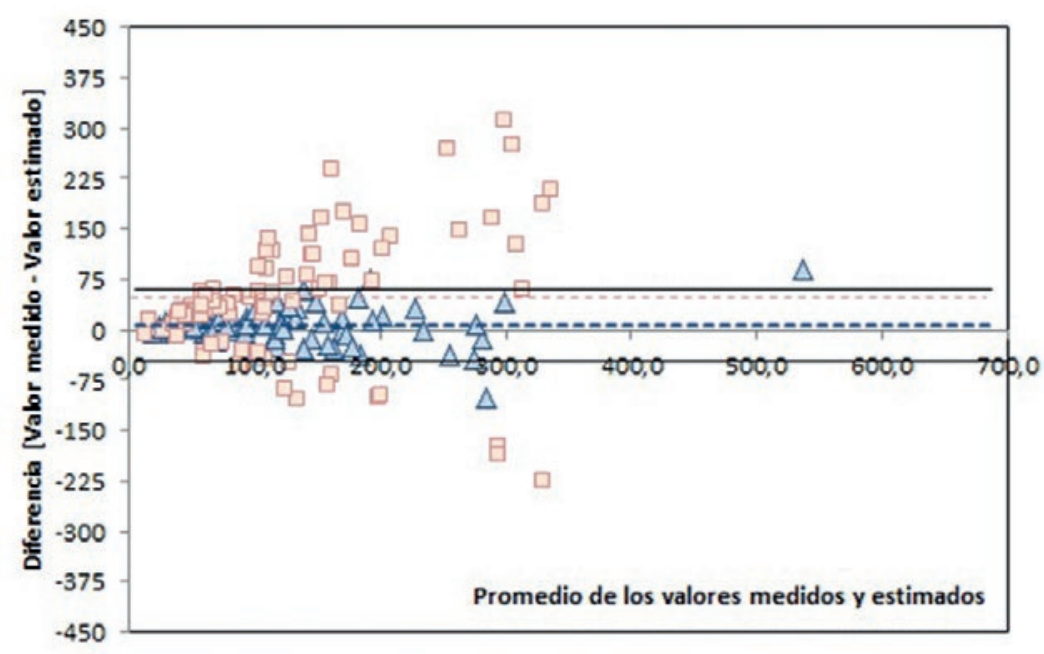

$\Delta$ Colecciones adecuadas $\square$ Colecciones inadecuadas

Figura 2. Gráfico de Bland-Altman para la concordancia entre la calciuria de 24 horas estimada mediante una fórmula propietaria y la determinada tras la recolección de orina de 24 horas. Las líneas continuas de color azul representan los límites de control al 95\% para la diferencia entre los valores estimados y medidos con las colecciones adecuadas de orina de 24 horas. Línea discontinua azul: promedio de las diferencias obtenidas con las colecciones adecuadas. Línea discontinua roja: promedio de las diferencias obtenidas con las colecciones inadecuadas.

en orina de 24 horas en todas las colecciones (medida $3.0 \pm 2.3 \mathrm{mg} / \mathrm{kg}$ en $24 \mathrm{~h}$ vs. estimada del ICaCre
$3.8 \pm 2.6 \mathrm{mg} / \mathrm{kg}$ en $24 \mathrm{~h}[\Delta=-0.8 ; \mathrm{p}<0.05 ;$ prueba de comparación de muestras apareadas basada en la 
Tabla 3. Concordancia diagnóstica entre los métodos de cálculo de la calciuria de 24 horas

\begin{tabular}{|l|c|c|c|}
\multirow{2}{*}{ Medidos } & \multicolumn{2}{|c|}{ Estimados } & \multirow{2}{*}{ Totales } \\
\cline { 2 - 3 } & $>\mathbf{4 . 0}$ & $\leq \mathbf{4 . 0}$ & \\
\hline$>4.0$ & 24 & 2 & 26 \\
\hline$\leq 4.0$ & 8 & 47 & 55 \\
\hline Totales & 32 & 49 & 81 \\
\hline
\end{tabular}

$\chi 2=3.6$ (prueba de McNemar; $p>0.05) ;$ kappa $=0.73(p<0.05)$. Fuente: registros del estudio.

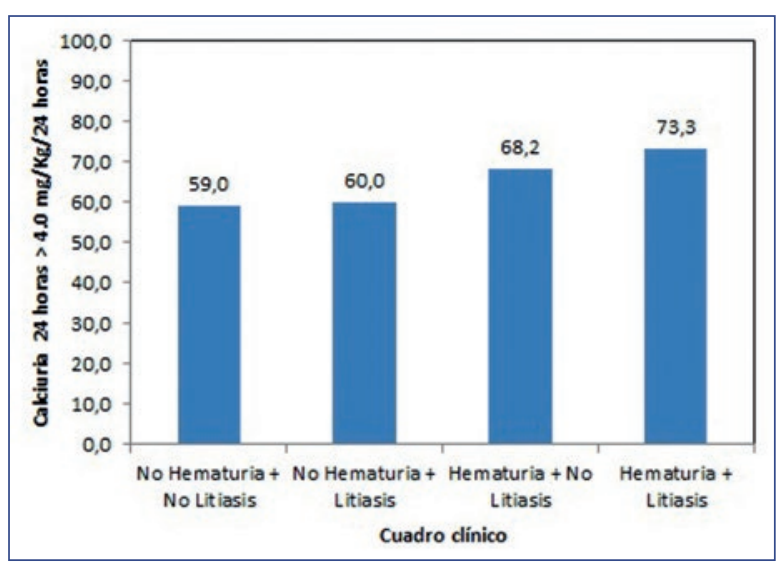

Figura 3. Distribución de la hipercalciuria entre los distintos síndromes clínicos encontrados en la serie. La calciuria de 24 horas fue estimada del índice calcio/ creatinina construido con una muestra matutina de orina una vez ajustado según la talla y la superficie corporal del niño 0 adolescente.

distribución $\mathrm{t}$ de Student]). Las menores diferencias entre los valores medidos y estimados de la calciuria de 24 horas se observaron con las colecciones adecuadas de orina de 24 horas (medida $3.6 \pm 2.3 \mathrm{mg} / \mathrm{kg}$ en $24 \mathrm{~h}$ vs. estimada del ICaCre $3.3 \pm 2.3 \mathrm{mg} / \mathrm{kg}$ en $24 \mathrm{~h}[\Delta=+0.3 ; \mathrm{p}<0.05$; prueba de comparación de muestras apareadas basada en la distribución $t$ de Student]), en comparación con las colecciones inadecuadas (medida $2.7 \pm 2.3 \mathrm{mg} / \mathrm{kg}$ en $24 \mathrm{~h}$ vs. estimada del ICaCre $4.0 \pm 2.9 \mathrm{mg} / \mathrm{kg}$ en $24 \mathrm{~h}[\Delta=-1.3 ; \mathrm{p}<0.05$; prueba de comparación de muestras apareadas basada en la distribución $t$ de Student]).

La figura 1 muestra la comparabilidad entre los valores observados y los predichos de la calciuria de 24 horas en el rango analítico de trabajo. Los valores observados de la calciuria de 24 horas se distribuyeron entre 6.6 y $493.4 \mathrm{mg}(0.1-12.0 \mathrm{mg} / \mathrm{kg})$. Cuando se recurrió a las colecciones adecuadas de orina de 24 horas, los estadígrafos de la recta de comparación de métodos fueron como sigue: coeficiente $r^{2}=0.91$; error típico de la regresión $=26.0$; intercepto $a=9.3$ $(p>0.05)$; pendiente $b=0.97(p<0.05)$. El uso de las colecciones inadecuadas de orina de 24 horas resultó en un incremento de la imprecisión intermétodo: coeficiente $r^{2}=0.29$ (diferencia respecto del obtenido con las colecciones adecuadas: $\Delta=-0.62)$; error típico de la regresión $=90.3(\Delta=+64.3)$; intercepto $a=79.8$ $(\Delta=+70.5)$; pendiente $b=0.69(\Delta=-0.28)$.

La figura 2 muestra la relación entre los valores medidos y estimados de la calciuria (absoluta) de 24 horas, tal como se refleja a través del gráfico de Bland-Altman. Las colecciones inadecuadas de orina introdujeron sesgos notables en el valor estimado de la calciuria de 24 horas: diferencia promedio, colecciones adecuadas $6.2 \pm 26 \mathrm{mg} / 24 \mathrm{~h}$ vs. colecciones inadecuadas $47.8 \pm$ $93.7 \mathrm{mg} / 24 \mathrm{~h}(\Delta=+41.6 ; \mathrm{p}<0.05$; prueba t de Student para la comparación de medias independientes). Las diferencias introducidas por la ecuación propietaria fueron del $22 \%$ en la calciuria medida en una colección adecuada de 24 horas. Estas llegaron a ser del 40\% cuando la colección fue inadecuada.

Los límites al 95\% para las diferencias entre la calciuria de 24 horas medida y estimada contuvieron el $95.1 \%$ de los resultados obtenidos con colecciones adecuadas de orina, pero solo el $18.6 \%$ de los obtenidos con colecciones inadecuadas.

La tabla 3 muestra la concordancia diagnóstica entre los valores observados y los predichos de la calciuria de 24 horas. La concordancia diagnóstica fue del $87.7 \%$ (prueba de Kappa $=0.73 ; p<0.05$ ). Las discordancias encontradas no alcanzaron significación estadística $\left(\chi^{2}=3.6 ; p>0.05\right.$; prueba de McNemar para muestras apareadas).

Finalmente, la figura 3 muestra la distribución, según la condición clínica del paciente, de la calciuria de 24 horas $>4.0 \mathrm{mg} / \mathrm{kg}$ estimada del ICaCre construido con la muestra matutina de orina mediante las ecuaciones propietarias desarrolladas. Así, la hipercalciuria se presentó en el $61.5 \%$ de los niños y adolescentes estudiados. De acuerdo con la condición clínica del paciente, la frecuencia de hipercalciuria se comportó como sigue: no hematuria + no litiasis: $59.0 \%$ (diferencia respecto del estimado con todas las causas: $\Delta=-2.5 \%$ ); no hematuria + litiasis: $60.0 \%(\Delta=-1.5 \%)$; hematuria + no litiasis: $68.2 \%(\Delta=+6.7 \%)$; y hematuria + litiasis: $73.3 \%(\Delta=+11.5 \%)$. 


\section{Discusión}

Este trabajo ha examinado la utilidad analítica, diagnóstica y clínica del ICaCre como una alternativa a la colección de orina de 24 horas en el estudio de la hipercalciuria acompañante de la litiasis renal. En un estudio previo se comprobó que el ICaCre, ajustado según la talla y la superficie corporal del niño o adolescente, puede servir para obtener estimados confiables y razonablemente exactos de la calciura de 24 horas $^{16}$. El uso del ICaCre permitiría también acortar el tiempo de latencia entre el pedido de análisis y el informe del resultado, a la vez que contribuye a que los procesos diagnósticos que incorporan la calciuria de 24 horas como descriptor sean más expeditos y completos $^{16}$.

Cuando se aseguran colecciones adecuadas de orina de 24 horas, el empleo del ICaCre puede ofrecer valores de la excreción urinaria de calcio con una equivalencia $>95 \%$ y una exactitud diagnóstica $>90 \%$. Además, el uso de este índice se distinguió por una concordancia diagnóstica $>85.0 \%$, lo que aseguraría la intercambiabilidad de los resultados emitidos con los métodos que han sido objeto de comparación.

Hasta el momento en que se realizó este estudio, el proceso diagnóstico de la litiasis renal ha descansado en la obtención de una colección de orina que sea representativa de un día en la vida del sujeto, para la determinación de la excreción renal de calcio. Son bien conocidas las insuficiencias de la determinación de la excreción urinaria de sustancias especificadas en colecciones de orina de 24 horas $^{24,25}$. Por consiguiente, cualquier alternativa que devuelva los valores exactos de la excreción urinaria de la sustancia de interés y resulte a la vez en una tasa superior de culminación del proceso diagnóstico será útil| ${ }^{26}$. La construcción del ICaCre con una muestra matutina de orina y su conversión en un estimado de la calciuria de 24 horas tras la corrección de acuerdo con las características antropométricas del niño o adolescente representaría tal alternativa, como ha quedado expuesto en este artículo. Investigaciones ulteriores se orientarían a evaluar el costo-efectividad de la introducción de las ecuaciones predictivas en la dinámica de un laboratorio, verticalizado en el estudio de la función renal.

El presente trabajo se ha extendido para evaluar la presencia de hipercalciuria en diferentes estados clínicos, como la hematuria no glomerular y la litiasis renal. Se debe señalar que en la institución de pertenencia de los autores actúa una consulta ambulatoria verticalizada en el diagnóstico y el tratamiento de la litiasis renal. La determinación de la excreción urinaria de calcio es entonces un elemento analítico clave dentro del protocolo hospitalario de diagnóstico de la litiasis renal. En tal sentido, en la serie de estudio construida para este ensayo, la frecuencia observada de hipercalciuria fue del $61.5 \%$.

La hipercalciuria es altamente prevalente en la nefrolitiasis ${ }^{27,28}$, pero en muchas ocasiones puede encontrarse en ausencia de una causa reconocible para ello. La hipercalciuria denominada idiopática pudiera afectar al $40.0 \%$ de los niños que concurren a un servicio hospitalario de nefrología con hematuria y litiasis ${ }^{4,5,29}$. Sin embargo, y en lo que pudiera constituir el hallazgo destacable de este trabajo, la hipercalciuria se presenta en casi las tres cuartas partes de los niños en los que concurren hematuria y litiasis renal, hallazgo que se sumaría a otros previamente publicados ${ }^{5,30}$. De esta manera, se justifica la inclusión del ICaCre en el protocolo diagnóstico de los síntomas nefrourológicos de la niñez y la adolescencia, como la hematuria glomerular y la litiasis renal.

\section{Adendum}

Una calculadora que ofrece los cálculos descritos en este trabajo está disponible en línea en http://nefrocalc. sarhugo.com. También se ofrece al lector interesado una aplicación para dispositivos móviles en http://nefrotoolbox.sarhugo.com/.

\section{Responsabilidades éticas}

Protección de personas y animales. Los autores declaran que para esta investigación no se han realizado experimentos en seres humanos ni en animales.

Confidencialidad de los datos. Los autores declaran que han seguido los protocolos de su centro de trabajo sobre la publicación de datos de pacientes.

Derecho a la privacidad y consentimiento informado. Los autores declaran que en este artículo no aparecen datos de pacientes.

\section{Conflicto de intereses}

Los autores declaran no tener ningún conflicto de intereses. 


\section{Bibliografía}

1. Srivastava T, Alon US. Pathophysiology of hypercalciuria in children. Pediatr Nephrol. 2007;22:1659-73.

2. Velásquez Forero F, Esparza M, Salas A, et al. Risk factors evaluation for urolithiasis among children. Bol Med Hosp Infant Mex. 2016;73:228-36.

3. Levy F, Adams-Huet B, Pak CY. Ambulatory evaluation of nephrolithiasis: an update of a 1980 protocol. Am J Med. 1995;98:50-9.

4. Polito C, La Manna A, Cioce F, et al. Clinical presentation and natura course of idiopathic hypercalciuria in children. Pediatr Nephrol. 2000 15:211-4.

5. Stapleton FB, for the Southwest Pediatric Nephrology Study Group. Idiopathic hypercalciuria: association with isolated hematuria and risk for urolithiasis in children. Kidney Int. 1990;37:807-11.

6. Velásquez Forero F, García P, Triffitt J, et al. Prostaglandin E1 increases in vivo and in vitro calcitriol biosynthesis in rabbits. Prostagl Leukotr Ess Fatty Acids. 2006;75:107-15.

7. Healy KA, Hubosky SG, Bagley DH. 24-hour urine collection in the metabolic evaluation of stone formers: is one study adequate? J Endourol. 2013;27:374-8.

8. Caleffi A, Manoni F, Alessio MG, et al. Quality in extra-analytical phases of urinanalysis. Biochem Med [Zagreb]. 2010;20:179-83.

9. Tormo C, Lumbreras B, Santos A, et al. Strategies for improving the collection of 24-hour urine for analysis in the clinical laboratory: redesigned instructions, opinion surveys, and application of reference change value to micturition. Arch Pathol Lab Med. 2009;133:1954-60.

10. Gokce C, Gokce G, Baydine C. Use of random urine samples to estimate total urinary calcium and phosphate excretion. Arch Intern Med. 1991;151:1587-8

11. Parekh DJ, Pope JC, Adams MC, et al. The association of an increased urinary calcium-to-creatinine ratio and asymptomatic gross and microscopic hematuria in children. J Urology. 2002;167:272-4.

12. Lavocat MP, Freycon MT, Muchrif M. Comparative study of 24-hour calciuria and urinary calcium/creatinine ratio in children over 4 years of age. Pediatrie. 1991;47:565-8

13. Sargent JD, Stukel TA, Kresel J, et al. Normal values for random urinary calcium to creatinine ratios in infancy. J Pediatr. 1993;123:393-7.

14. Koyun M, Güven AG, Filiz S, et al. Screening for hypercalciuria in schoolchildren: what should be the criteria for diagnosis? Pediatr Nephrol. 2007;22:1297-301.
15. Rath B, Aggarwal MK, Mishra TK, et al. Urinary calcium creatinine ratio and hypercalciuria. Indian Pediatr. 1994;31:311-6.

16. Salabarría González JR, Santana Porbén S, Ricabal MDRL. Excreción urinaria de una sustancia predicha del índice sustancia-creatinina. Rev Latinoam Patol Clin Med Lab. 2015;62:119-26.

17. Santana Porbén S. Herramientas informáticas para el cálculo de los indicadores de la función renal. En: Salabarría González JR, Santana Porbén S, editores. Laboratorio clínico y función renal. Madrid: Editorial EAE Académica Española; 2011.

18. Bartels H, Cikes M. Ueber Chromogene der Kreatininbestimmung Nach Jaffé. [Chromogens in the creatinine determination of Jaffé]. Clin Chim Acta. 1969;26:1-10.

19. Michalylova V, Ilkova P. Photometric determination of micro-amounts of calcium with arsenazo III. Anal Chim Acta. 1971;:53:194-8.

20. Delafield DuBois BS, DuBois EF. The measurement of the surface area of man [Fifth paper in a series]. Arch Intern Med (Chic). 1915;15:868-81.

21. Ghazali S, Barratt TM. Urinary excretion of calcium and magnesium in children. Arch Dis Child. 1974;49:97-101.

22. Counahan R, Chantler C, Ghazali S, et al. Estimation of glomerular filtration rate from plasma creatinine concentration in children. Arch Dis Child. 1976;51:875-8.

23. Santana Porbén S, Martínez Canalejo H. Manual de procedimientos bioestadísticos. Madrid: EAE Editorial Académica Española; 2012.

24. Ulmann A. Predictive value of lithogenic risk in hypercalciuria: should 24-hour urine calcium be measured? Nephrologie. 1983;5:232-4.

25. Riess C, Hess B, Binswanger U. Questionable significance of the chemical analysis of a single 24-hour urine sample in recurrent calcium oxalate nephrolithiasis. Klinische Wochenschrift. 1986;64:411-6.

26. Ring $E$, Borkenstein $M$. Use of the calcium-creatinine ratio in diagnosis and therapy. Padiatr Padol. 1987;22:245-50

27. Matsushita K, Tanikawa K. Significance of the calcium to creatinine concentration ratio of a single-voided urine specimen in patients with hypercalciuric urolithiasis. Tokai J Exp Clin Med. 1987;12:167-71.

28. Milliner DA. Urolithiasis. En: Avner ED, Harmon WE, Niaudet $P$, editores. Pediatric nephrology. Philadelphia: Lippincott Williams \& Wilkins; 2004. p. 1091-111.

29. Escribano J, Balaguer A, Martín R, et al. Childhood idiopathic hypercalciuria. Scand J Urol Nephrol. 2004;38:422-6.

30. Stapleton FB, Roy III S, Noe HN, et al. Hypercalciuria in children with hematuria. N Engl J Med.1984;310:1345-8. 